\title{
Bladder Tumor Recurrence after Primary Surgery for Transitional Cell Carcinoma of the Upper Urinary Tract
}

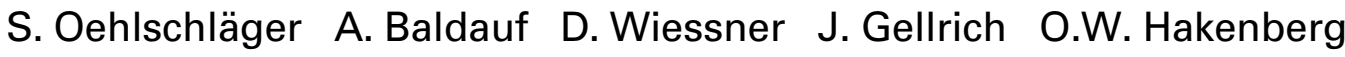 \\ M.P. Wirth
}

Department of Urology, Technical University Dresden, Dresden, Germany

\section{Key Words}

Transitional cell carcinoma - Upper urinary tract . Bladder tumor recurrence

\begin{abstract}
Objective: Primary transitional cell carcinoma (TCC) of the upper urinary tract represents $6-8 \%$ of all TCC cases. Nephroureterectomy with removal of a bladder cuff is the treatment of choice. The rates of TCC recurrence in the bladder after primary upper urinary tract surgery described in the literature range between 12.5 and $37.5 \%$. In a retrospective analysis we examined the occurrence of TCC after nephroureterectomy for upper tract TCC in patients without a previous history of bladder TCC at the time of surgery. Methods: Between 1990 and 2002, 29 patients underwent primary nephroureterectomy for upper tract TCC. The mean age of the patients was 69.5 years. In 5 cases upper urinary tract tumors were multilocular, in the remaining cases unilocular in the renal pelvis $(n=12)$ or the ureter $(n=12)$. The followup was available for 29 patients with a mean follow-up of 3.37 (0.1-11.2) years. Results: $11 / 29$ (37.9\%) patients had TCC recurrence with 9/11 patients having bladder TCC diagnosed within 2.5 years $(0.9-6.0)$ after nephroureterectomy. 13/29 patients are alive without TCC recurrence,
\end{abstract}

3/29 patients died due to systemic TCC progression and $5 / 29$ died of unrelated causes without evidence of TCC recurrence. Conclusion: Our data indicate a high incidence of bladder TCC after nephroureterectomy for primary upper tract TCC of up to 6 years after primary surgery. Because of the high incidence of bladder TCC within the first 3 years of surgery, careful follow-up is needed over at least this period.

Copyright $@ 2004$ S. Karger AG, Base

\section{Introduction}

The primary diagnosis of upper urinary transitional cell carcinoma (TCC) accounts for approximately 6-8\% of all TCCs [1]. The treatment of choice for upper tract TCC is nephroureterectomy with the removal of a bladder cuff. Recurrence rates after nephroureterectomy given in the literature range from 12.5 to $38 \%$ [2-4].

In case of contraindications to nephrectomy (azotemia, solitary kidney, bilateral disease) renal-sparing surgery is advocated [5], which includes open segmental and endoscopic procedures. In the literature, segmental ureteral resection has been preferred for low-grade and lowstage tumors $[1,5]$.

\begin{tabular}{ll}
\hline KARGER & ( ) 2004 S. Karger AG, Basel \\
Fax +41642-1138/04/0733-0209\$21.00/0 \\
$\begin{array}{l}\text { E-Mail karger@ 12 34 karger.ch } \\
\text { www.karger.com }\end{array}$ & $\begin{array}{l}\text { Accessible online at: } \\
\text { www.karger.com/uin }\end{array}$
\end{tabular}

OA Dr. med. S. Oehlschläger

Klinik für Urologie, Universitätsklinikum Carl-Gustav-Carus

Fetscherstrasse 74, DE-01307 Dresden (Germany)

Tel. +49 3514582447 , Fax +49 3514584333

E-Mail sven.oehlschlaeger@mailbox.tu-dresden.de 
Charbit et al. [6] calculated a risk of bladder TCC recurrence of approximately $9 \%$ after upper tract TCC surgery. In patients with a previous history of bladder TCC, a higher risk of bladder TCC recurrence up to 70\% has been reported [6, 7]. Krogh et al. [1] reported a bladder TCC recurrence rate of $36.4 \%$ after upper tract TCC surgery. However, in their retrospective series, 198 patients with different and not comparable surgical techniques as well as patients with concurrent as well as preceding bladder TCCs at the time of nephroureterectomy were included.

Because of the varying recurrence rates described in the literature, we analyzed the bladder TCC recurrence rate after nephroureterectomy for TCC of the upper tract in the subgroup of patients without concurrent or previous bladder TCC at the time of surgery of nephroureterectomy.

\section{Materials and Methods}

Between 1990 and 2002, 29 patients (21 men and 8 women) without cystoscopic evidence of concurrent bladder TCC and without a history of previous bladder TCC underwent primary nephroureterectomy. The TCC was located on the right side in 15 patients and on the left side in 14 patients. 26 patients underwent open nephroureterectomy with open bladder cuff resection. Laparoscopic nephrectomy with open ureterectomy and bladder cuff resection was done in 3 patients. The mean age of the patients was 69.5 (39.9-84.3) years. In 5 cases the upper urinary tract tumors were multilocular in the renal pelvis and the ureter, in 12 cases unilocular in the renal pelvis and in 12 cases unilocular in the ureter. The pathologic stage was pTa,N0,M0,R0,G2 in 1 patient, pT1,N0,M0,R0,G1-2 in 9 patients, pT2,N0,M0,R0,G2-3 in 11 patients, pT3,N0,M0,R0,G2-3 in 6 patients. Resection of the bladder cuff was histologically incomplete (R1) (pT3,N0,M0,R1,G3) in 2 cases.

Tumor recurrence was detected by abdominal sonography, urine cytology, cystoscopy and/or intravenous pyelography. Follow-up was done by office urologists. For statistical analysis, cross tables with $\chi^{2}$ test and the Pearson correlation were used.

\section{Results}

Mean follow-up was $3.37(0.1-11.2)$ years. The 2 cases with R1 status after nephroureterectomy had had histologically incomplete resection of TCC at the bladder cuff. One patient with pT3 status and positive margins at the bladder cuff received adjuvant chemotherapy without tumor recurrence. The other patient with pT3 R1 status died 7 months after surgery aged 85 without evidence of recurrence. $3 / 29(10.3 \%)$ patients died due to tumor progression and $5(17.2 \%)$ died of unrelated causes. $2 / 29$
(6.9\%) patients after removal of pT2-3,N0,M0,R0,G2-3 unilocular upper tract TCC developed hepatic and pulmonary metastases or localized TCC of the contralateral ureter without bladder TCC after 6 and 18 months, respectively. Both patients received systemic palliative chemotherapy and died due to tumor progression.

11/29 (37.9\%) patients suffered TCC recurrence overall. In 9/29 (31.0\%) patients bladder TCC was diagnosed in a mean time of 2.5 (range 0.9-6.0) years after surgery. Overall, 6/9 patients with bladder TCCs recurrence developed bladder TCC within the first 3 years after nephroureterectomy. Only $1 / 9$ patients had bladder TCC more than 5 years after nephroureterectomy. While none of these patients suffered from muscle-invasive bladder TCC, 3/9 patients had a high-grade bladder TCC. Two patients with high-grade bladder TCC underwent radical cystectomy in a mean time of 1 year after nephroureterectomy. In 1 case the bladder TCC was seen in combination with systemic progression. Despite systemic chemotherapy, the recurrent TCC led to death of disease 3.9 years after primary surgery.

In our study, male patients had a significant higher risk of bladder TCC recurrence $(\mathrm{p}<0.02)$. We found no correlations between stage $(p=0.056)$, grade $(p=0.095)$ or multifocality $(\mathrm{p}=0.726)$ in the upper urinary tract and the incidence of bladder TCC recurrence. 13/29 (44.8\%) patients are alive without tumor recurrence with a mean follow-up of 4.32 (range $0.49-11.2$ ) years.

\section{Discussion}

TCC of the upper tract occurs in older patients $[1,8]$. The mean age of our patients was 69.5 years and 16/29 $(55.2 \%)$ patients in our series were over 70 years old. Nephroureterectomy with a bladder cuff is the standard treatment for upper tract TCC because of the multifocal nature of this malignancy. An evaluation of the influence of using an open versus an endoscopic technique for bladder cuff removal on the rate of bladder TCC recurrence after nephroureterectomy in 145 patients showed no significant difference in the recurrence rate [8]. In selected cases with contraindications of nephrectomy, nephronsparing surgery is recommended for single, low-grade and stage TCC in the distal ureter. The survival rates of patients who underwent partial ureterectomy or complete nephroureterectomy were equal for low-grade and stage TCC [9].

In our series all patients were preoperatively free of bladder TCC on cystoscopy. 9/29 (31.0\%) of this selected 
group of patients later developed TCC recurrence in the bladder. This is comparable to the data in the literature which, however, include several series with patients included with a previous history of bladder TCC $[1,6-8,10$, 11]. Therefore, our data with a selected group of patients without a history of previous bladder TCC and without concurrent bladder TCC give a reasonable indication of the true risk of bladder TCC recurrence after surgery for upper tract TCC.

In $6 / 9(66.7 \%)$ of the patients with bladder TCC recurrence, the bladder tumor occurred within the first 3 years, with a mean time of 2.5 years. Patients with high-grade bladder TCC recurrence required radical cystectomy within the first 12 months after nephroureterectomy. Patients with systemic recurrence of TCC were detected within the first 2 years.

Various possible risk factors for the development of bladder TCC recurrence have been described in the literature. Independent factors for bladder TCC recurrence seem to be multifocality of the upper tract TCC and pathologic stage [7, 10]. Salvador-Bayarri et al. [8] described a higher incidence of bladder recurrence after high-grade upper tract TCC, while Koga et al. [11] in their series described female sex, the administration of adjuvant chemotherapy and incomplete distal ureterectomy (R1) as risk factors for the bladder TCC recurrence after nephroureterectomy for upper tract TCC. In contrast to the literature, we found correlations between the male sex and bladder TCC recurrence in our series. The stage $(\mathrm{p}=$ $0.056)$ and the grade $(p=0.095)$ of the upper tract TCC correlated without statistical significance. This may possibly be related to the high number of low-stage and lowgrade upper tract TCCs in our series and possibly also to the lack of statistical power due to the small number of patients with TCC recurrence in this highly selected group.

\section{Conclusions}

Our data show a high incidence of bladder TCC recurrence after nephroureterectomy for TCC of the upper tract of up to 6 years after primary surgery. Because of the high incidence of bladder tumor recurrence appearing within the first 3 years after surgery, a careful follow-up is mandatory over at least this period.

\section{References}

1 Krogh J, Kvist E, Rye B: Transitional cell carcinoma of the upper urinary tract: Prognostic variables and post-operative recurrences. $\mathrm{Br} \mathrm{J}$ Urol 1991;67:32-36.

2 Laguna MP, de la Rosette JJ: The endoscopic approach to the distal ureter in nephroureterectomy for upper urinary tract tumor. J Urol 2001;166:2017-2022.

3 Mufti GR, Gove JR, Badenoch DF, Fowler CG, Tiptaft RC, England HR, Paris AM, Singh M, Hall MH, Blandy JP: Transitional cell carcinoma of the renal pelvis and ureter. Br J Urol 1989;63:135-140.

4 Murphy DM, Zincke H, Furlow WL: Management of high grade transitional cell cancer of the upper urinary tract. J Urol 1981;125:2529.
5 Canfield SE, Dinney CP, Droller MJ: Surveillance and management of recurrence for upper tract transitional cell carcinoma. Urol Clin North Am 2003;30:791-802.

6 Charbit L, Gendreau MC, Mee S, Cukier J: Tumors of the upper urinary tract: 10 years of experience. J Urol 1991;146:1243-1246.

7 Sakamoto N, Naito S, Kotoh S, Nakashima M, Nakamura M, Ueda T, Kumazawa J: Recurrence of bladder tumors following surgery for transitional cell carcinoma of the upper urinary tract. Eur Urol 1991;20:136-139.

8 Salvador-Bayarri J, Rodriguez-Villamil L, Imperatore V, Palou Redorta J, VillavicencioMavrich H, Vicente-Rodriguez J: Bladder neoplasms after nephroureterectomy: Does the surgery of the lower ureter, transurethral resection or open surgery, influence the evolution? Eur Urol 2002;41:30-33.
9 Murphy DM, Zincke H, Furlow WL: Primary grade 1 transitional cell carcinoma of the renal pelvis and ureter. J Urol 1980;123:629-631.

10 Hisataki T, Miyao N, Masumori N, Takahashi A, Sasai M, Yanase M, Itoh N, Tsukamoto T: Risk factors for the development of bladder cancer after upper tract urothelial cancer. Urology 2000;55:663-667.

11 Koga F, Nagamatsu H, Ishimaru H, Mizuo T, Yoshida K: Risk factors for the development of bladder transitional cell carcinoma following surgery for transitional cell carcinoma of the upper urinary tract. Urol Int 2001;67:135141. 